\title{
VIETNAM 2018
}

\author{
Maricela Mireya Reyes López \\ Universidad de Colima
}

\section{INTRODUCCIÓN}

La República Socialista de Vietnam (nombre oficial de Vietnam), transitó por la senda del reconocimiento político internacional luego de haber sido anfitrión en la reunión de líderes del Foro de Cooperación Económica Asia-Pacífico (APEC, por sus siglas en inglés) a finales de 2017, y del Foro Económico Mundial de la Asociación de Naciones del Sudeste Asiático (ASEAN, por sus siglas en inglés) en 2018. No obstante, su talón de Aquiles es el desarrollo del conflicto regional del Mar del Este, como lo llama Vietnam, o Mar del Sur de China, como lo llama China. ${ }^{1}$ Este conflicto, recurrente y con pocas probabilidades de solución, tiene de algún modo probabilidades de ser contenido. El desempeño de Vietnam en cuanto al reclamo de las islas Paracel, y el apoyo estadounidense, ponen al país en una situación difícil frente a su vecino del norte, China, al que la diplomacia de las partes involucradas debería de convencer para firmar compromisos de conducta.

En el ámbito nacional, Vietnam ha sido merecedor del reconocimiento internacional, sobre todo en el aspecto macroeconómico. Un crecimiento económico sostenido, pronosticado en $6.8 \%$ para finales de 2018, un desarrollo económico positivo, y la atracción de inversiones, son los pilares de la buena economía que, hasta cierto punto, le han elogiado instituciones financieras regionales. Durante tres décadas, los graduales índices positivos se han reflejado en el alfabetismo, el empleo, disminución de la pobreza, esperanza de vida y

\footnotetext{
${ }^{1}$ En este texto se utilizará Mar del Sur de China, por ser el más utilizado y porque no genera confusión geográfica.
} 
desarrollo humano. De los países comunistas del mundo, Vietnam es ejemplo por su prosperidad en desarrollo tecnológico e infraestructura, y en competitividad. También es considerado poseedor de una de las economías de ingreso medio de mayor crecimiento sostenido en el sureste de Asia.

Sus logros pudieron verse afectados debido a la inestabilidad económica, por lo que tuvo que fortalecer y generar confianza en asuntos como: la firma de acuerdos de libre comercio (TLC) con países claves, el Acuerdo Integral y Progresivo para la Asociación Transpacífico (СРTPP, por sus siglas en inglés), y el Acuerdo de Libre Comercio VietnamUnión Europea (EVFTA, por sus siglas en inglés).

Las siguientes referencias hacen alusión a los principales hechos de 2018 que marcaron el devenir de la política internacional en Vietnam, su política exterior, así como los intentos por fortalecer sus relaciones con otros países de Asia-Pacífico, además del quehacer económico interno.

\section{POLÍTICA INTERNACIONAL}

Sin duda alguna el quehacer político de la administración estadounidense, o mejor dicho las decisiones imprevistas del presidente Donald Trump, permearon la toma de decisiones de los gobiernos del sureste de Asia, y Vietnam no fue la excepción. Dos temas en este sentido son el conflicto recurrente del Mar del Sur de China y el recién nombrado Acuerdo Integral y Progresivo para la Asociación Transpacífico.

\section{El eterno conflicto en el Mar del Este/Mar del Sur de China}

El tema de reclamo territorial en el Mar del Sur de China por parte de países del sureste de Asia, incluido Vietnam (y de las Paracel por parte de Taiwán), representa para éste la incertidumbre de aceptar abiertamente a Estados Unidos como su aliado, o desconfiar de las acciones chinas. En enero de 2018 James Mattis, secretario de Defensa de Estados Unidos, declaró, cuando se encontraba de visita oficial en Vietnam, que para la Casa Blanca era importante la relación de asociación integral Vietnam-Estados Unidos en todas las ramas, incluidas seguridad y defensa (VietnamPlus, 2018). Dos meses después de esa declaración, un portaaviones estadounidense, el Carl Vinson, atracó en la costa vietnamita de Cam Ranh, 
frente a la provincia de Danang. La asociación integral Vietnam-Estados se tradujo así en la presencia militar estadounidense, por primera vez en territorio vietnamita desde que terminó la guerra de 1975.

No debe menospreciarse este hecho porque el portaaviones que atracó llevaba 72 naves y 5500 soldados (Beech, 2018). El portaaviones Carl Vinson es uno de los cinco más grandes del mundo; los otros pertenecen a China, India, Francia y Rusia.

Una segunda presencia militar estadounidense en territorio vietnamita se dio dos meses después, cuando dos buques de guerra realizaron maniobras mediante el esquema Libertad de Navegación (Fonop, por sus siglas en inglés), dentro de las 12 millas náuticas de islotes que ocupa China y que directamente reclama Vietnam. Los islotes, Tree, Triton, Lincoln y Woody, forman parte de las islas Paracel. Las maniobras estadounidenses tuvieron por objetivo ampliar el alcance y la frecuencia de las Fonop en el Mar del Sur de China. En esa ocasión los buques de guerra de la marina de Estados Unidos que maniobraron en dichas aguas fueron un destructor clase Arleigh Burke, el USS Higgins, y el crucero USS Antietam. Dichos buques navegaron dentro de las 12 millas náuticas, en las islas Paracel. La prensa vietnamita consideró las acciones como evidencia del compromiso de Estados Unidos con Vietnam.

Algunos analistas opinan que para Estados Unidos Vietnam es el socio ideal (y, francamente, el único), dispuesto a enfrentar a China por sus reclamos en esta disputa territorial. También hay posiciones diferentes en este asunto, como la del especialista en el Mar del Sur de China y el papel de Vietnam, Carl Thayer, quien argumenta que el arribo del Carl Vinson representa una evolución en el compromiso militar entre Vietnam y Estados Unidos, pero que no significa que Hanoi se haya unido a Washington para oponerse a Beijing (Dang, 2018).

Durante la administración de Donald Trump, la marina de Estados Unidos ha aumentado la frecuencia de las Fonop, y no todas las operaciones se han reportado (Panda, 2018). Una declaración emitida por el Ministerio de Defensa chino condenó la operación, afirmando que había contravenido el derecho internacional chino, que infringió seriamente la soberanía china y que perjudicó la confianza estratégica mutua entre los dos ejércitos (Panda, 2018). Es obvio que las partes en conflicto se sienten ofendidas por igual. 
El arribo del portaaviones y las operaciones del Fonop en mayo significaron un desafío por parte Vietnam y EUA frente a una China que también responde. Por ello, como una estrategia por el dominio marítimo y aéreo, China está construyendo islas artificiales sobre cinco arrecifes en el Mar del Sur de China. Su respuesta ante el primer arribo fue realizar una misión de patrullaje, y la intención de llevar a cabo ejercicios de combate al día siguiente, según el anuncio de un funcionario militar de la fuerza aérea del Ejército de Liberación Popular chino. A decir de agencias de noticias internacionales, las acciones se efectuaron del 5 al 11 de abril (Gao, 2018).

Paralelamente, pero no como coincidencia, el ministro de Relaciones Exteriores chino, que se encontraba de visita oficial en Vietnam para asistir a la Reunión de Líderes de la Cooperación Económica de la Subregión del Gran Mekong (GMS, por sus siglas en inglés), no pudo dejar de mencionar el asunto de los reclamos y las acciones militares.

Sobre el mismo asunto, el 19 y 20 de junio, en el Departamento de Estado, en Washington D.C., se organizó el quinto diálogo entre EUA y Vietnam sobre asuntos marítimos y derecho del mar. Expertos en agencias marítimas y en asuntos exteriores estadounidenses y vietnamitas intercambiaron puntos de vista sobre cuestiones relacionadas con los océanos, el derecho al mar, así como sobre la cooperación marítima en foros internacionales y regionales. Las delegaciones de ambos países acordaron profundizar en el diálogo en estos temas en futuras reuniones (State, 2018). El gobierno vietnamita dejó entrever la posibilidad de organizar la siguiente ronda de expertos en su territorio, en 2019.

Como antesala del diálogo, la embajadora vietnamita ante la ONU, Nguyen Phuong Nga, durante la conferencia de integrantes de la Convención de las Naciones Unidas sobre el Derecho del Mar, de 1982, reiteró que su país desea la solución del conflicto, y elogió los esfuerzos de otros países por mejorar el código de conductas de las partes involucradas en esa diferencia (vov5, 2018).

\section{Vietnam mantiene interés en el Acuerdo Transpacifico}

El 8 de marzo de 2018 fue rebautizado en Chile el Acuerdo de Asociación Transpacífico (TPP por sus siglas en inglés, o TPP-12) con el nombre de Tratado Integral y Progresista de Asociación Transpacífico (CPTPP), con la firma de once integrantes y la ausencia de EUA (antes miembro del TPP). El CPTPP busca promover el crecimiento económico, crear empleos, 
reducir la pobreza y mejorar los niveles de vida de los pobladores de los países miembros. El rechazo de Donald Trump refiere, en principio, a que los acuerdos bilaterales son más eficientes que los multilaterales. El 13 de septiembre los gobiernos de Vietnam y Japón reiteraron la invitación a Estados Unidos a unirse al CPTTP, pero el país norteamericano sigue sin ofrecer respuesta.

Para Vietnam y para todos los miembros, el CPTPP representa participar en uno de los bloques más dinámicos de libre comercio en el mundo, con un mercado combinado de alrededor de 499 millones de personas y un Producto Interno Bruto (PIB) total de 10.1 billones de dólares, que representan el 13.5\% del PIB global (Vietnam, 2018).

El interés de Vietnam en participar en el CPTPP es porque busca diversificar su economía mediante la participación en diversos espacios transpacíficos, ya que necesita depender menos de China y al mismo tiempo ampliar su mercado con EUA. Asimismo, las exportaciones vietnamitas se verían fortalecidas con mercados amplios, como el de Japón, Australia, Canadá y México, quienes son miembros del CPTPP.

En opinión del Banco Mundial, Vietnam sería uno de los miembros más beneficiados del CPTPP, puesto que, según supuestos conservadores, en 2030 el impacto económico del acuerdo en Vietnam sería un aumento en el PIB, crecimiento en importaciones y exportaciones, menores tarifas a sus exportaciones y mejoras en el volumen de sus sectores de alimentos, bebidas, tabaco, ropa, cuero y textiles; con un crecimiento más modesto en los subsectores manufacturero y de servicios. Se predice que las importaciones crecerán en todos los sectores (Maliszewska, Olekseyuk y Osorio-Rodarte, 2018).

Actualmente Vietnam es integrante de 16 acuerdos de libre comercio, incluyendo a los más grandes de Asia, uno con China y otro con Japón, y pretende lograr una amplia apertura mercantil con EUA. La relación económica actual Vietnam-Estados Unidos presenta comercio bilateral en aumento, comparativamente de 1995 a 2015, de 451 millones de dólares a 45 mil millones, respectivamente. En realidad Vietnam debe diversificar a sus socios comerciales, porque tiene un gran déficit con China en este tema (Maliszewska, Olekseyuk y Osorio-Rodarte, 2018).

El interés vietnamita en el CPTPP no es sólo económico y comercial, toda vez que los liberales locales aplauden este tratado porque obliga a Vietnam a adherirse a normas 
internacionales de responsabilidad laboral y gubernamental que el gobierno de Hanoi no podría cumplir. Aunque son países comunistas China y Vietnam, no todos en Vietnam quieren desarrollarse de la misma manera como lo hace China, esto es, no obstante que el mercado de exportaciones vietnamitas está dirigido en su mayoría a Estados Unidos, el mayor socio comercial es China, con quien también tiene un enorme déficit comercial. En suma, el interés vietnamita en el CPTPP es también geopolítico y social, como lo señala Nguyen Ngoc Anh, profesora de la Universidad de Comercio Exterior de Hanoi (Beech, 2017).

En otro asunto, vinculado con los temas regionales y de impacto internacional, Vietnam fue anfitrión del Foro Económico Mundial de la ASEAN (FEM-ASEAN 2018), del 11 al 13 de septiembre. Los temas ahí tratados fueron la innovación y los retos para el empleo de la llamada cuarta revolución industrial. A este evento asistieron líderes de gobiernos, empresas y personas de todas las nacionalidades, miembros de la ASEAN.

Las discusiones giraron en torno al título del evento "ASEAN 4.0: Espíritu emprendedor y la cuarta revolución industrial", en temáticas como competencia global; pequeñas, medianas y nuevas empresas; tecnología digital; economía moderna; automatización; inteligencia artificial; robots; dispositivos satelitales; vehículos aéreos no tripulados; sistemas sensoriales para productividad; envejecimiento de la población; economía de internet; agricultura de alta tecnología, y otros (incluidos temas controversiales como Mar del Sur de China y desnuclearización de Corea del Norte). La variedad y novedad de los temas justifica que este evento haya contado con aproximadamente mil participantes de 43 países, entre jefes de Estado y de gobierno de la ASEAN.

Vietnam propuso la expansión del modelo de redes móviles con precio único entre Camboya, Laos, Myanmar y Vietnam, para que las personas puedan tener mejores servicios de telecomunicaciones a bajo precio; por ejemplo, los servicios de telefonía móvil entre países pueden tener un precio único y beneficiarse si los nacionales estuvieran fuera de sus países. Entre otras ideas, también propuso la cooperación en inteligencia, mantenimiento de la seguridad cibernética, capacitación de personal y tecnología de la información. El evento volvió a posicionar a Vietnam en la región como un actor diplomático de confianza (al igual que en la reunión de la APEC). 


\section{Política exterior más dinámica}

La política exterior de Vietnam se basa en lograr la integración del país lo más ampliamente posible; por ello, su tarea de estrechar relaciones es imperante, lo que demuestra la serie de visitas a varios países realizadas por el primer ministro, Nguyen Xuan Phuc, y el secretario general del Partido Comunista de Vietnam, Nguyen Phu Trong, así como por funcionarios de alto nivel.

\section{Vietnam y Estados Unidos}

Uno de los antecedentes de la actual política exterior de Vietnam con Estados Unidos fue la visita del primer ministro de Vietnam, Nguyen Xuan Phuc, a tierra norteamericana en mayo de 2017. Fue el primer mandatario del sureste de Asia que se entrevistó con Donald Trump, en un acontecimiento especial porque casi siempre se relaciona a estos dos países con la guerra. Aquella visita fue incierta y no ocurrió en el mejor momento de la relación, puesto que el presidente estadounidense no había expresado interés por el sureste de Asia, ni mucho menos por Vietnam.

¿Cuál fue entonces la intención de esa visita?: fortalecer la relación diplomática y comercial en el contexto de la incertidumbre provocada por la salida de EUA del TPP, y algunos resultados lo evidencian en 2018. Vietnam desea fortalecer lazos con EUA para no quedar al margen de aquello que se hubiera preparado. Asimismo, es posible que al crear una nueva relación con EUA, Vietnam puede equilibrar la dependencia que tiene con China. De igual manera, es importante para Vietnam que Donald Trump acepte un comercio más abierto, y difuminar la imagen proteccionista que lo caracteriza. A Vietnam le interesa atraer inversión extranjera directa (IED) estadounidense y asegurar el acceso a su gran mercado.

No obstante, las tensiones comerciales entre EUA y China pueden resultar positivas para la economía vietnamita, pues una guerra comercial le favorecería si la IED fluyera hacia este país, en caso de que ya no fluyera hacia China; pero sólo si las empresas multilaterales diversificaran sus cadenas de suministro lejos de China. Por ejemplo, en lugar de producir partes y componentes de productos electrónicos en países del sureste asiático y de hacer el montaje final en China, las compañías multinacionales podrían trasladar sus operaciones a 
países vecinos (Suiwah, 2018); en ese momento Vietnam sería el país más atractivo y se vería favorecido con la inversión extranjera directa.

Otra visita destacada fue la que realizó el secretario de Estado norteamericano Michael Pompeo a Vietnam en julio de 2018, visita que formó parte de una gira diplomática que se extendió hacia Corea del Norte y Japón. Por decirlo de alguna manera, fue una visita cordial informal, pues Pompeo habló de Corea del Norte poniendo como ejemplo al país anfitrión, Vietnam. Pompeo elogió la economía de Vietnam en los siguientes términos: “[...] a communist nation and former foe can become a key American ally. Vietnam is now arguably America's closest partner in Southeast Asia" (Hutt, 2018). Dicha visita ocurrió enmedio de protestas por parte de activistas y defensores de derechos humanos.

\section{Vietnam y China}

Además del eterno conflicto territorial en el Mar del Sur de China, el tema trae aparejados otros conflictos domésticos que vulneran la relación entre Vietnam y China. Destaca la visita del secretario general del Partido Comunista de Vietnam a China el 12 de enero, con el objetivo de continuar la relación rota desde el incidente de la HYSY- $98,{ }^{2}$ plataforma de perforación petrolera que realizó operaciones en aguas disputadas dentro de la zona económica especial (ZEE) de Vietnam, por lo que este país respondió enviando buques de la Guardia Costera y de la Fuerza de Vigilancia Pesquera para protestar contra la violación china de su jurisdicción soberana. Aunque el incidente no pasó a mayores porque China se retiró, pero sí creó desconfianza y debilitó el vínculo de la relación. Ha resultado espinoso fortalecer la relación porque no cesan las protestas contra personas de origen chino.

Varias protestas de vietnamitas contra negocios o acciones comerciales de empresarios chinos se desataron por todo el país. Las principales fueron para mostrar inconformidad por la creación de ZEE, con el argumento de generar inversiones y reformas económicas. El asunto es que la nueva ley establece la entrega de tierras a inversionistas, en este caso chinos, con la modalidad de contratos de arrendamiento por 99 años. Los manifestantes determinaron

${ }^{2}$ Fue una crisis diplomática entre China y Vietnam en 2014, resultado de que el primero colocó una plataforma petrolera cerca de las islas Paracel, pertenecientes a Vietnam. La plataforma se llama Haiyang 981. Se produjeron protestas por parte de vietnamitas para impedir la instalación de plataformas. 
que no había zona especial y que no se arrendarían tierras a China, ni por un día (Fawthrop, 2018).

Ante la cada vez mayor posibilidad de que las cosas se salieran de control, porque iban creciendo por todo el país, el primer ministro dio marcha atrás a esta ley. Al principio hubo manifestaciones en Hanoi y Ho Chi Minh, que después se extendieron a ciudades en seis provincias, incluyendo Danang (sede de la APEC en 2017), Nha Trang, Binh Thuan y Tai Ninh. El asunto puede aún desatar una inestabilidad mayor, porque lo han apoyado intelectuales, académicos, e incluso funcionarios de gobierno (Fawthrop, 2018).

No deja de incomodar el problema entre la población vietnamita, que teme por las dos ZEE propuestas: una sugerida en Van Don, provincia de Quang Ninh, situada cerca de la frontera con China, y otra en la isla de Phu Quoc, provincia de Kien Giang, situada frente a la zona costera de Camboya, cada vez más dominada por proyectos chinos.

Puede decirse que la relación entre China y Vietnam no es todavía de carácter amistoso y confiable, por lo que la promesa hecha entre ambos líderes, en el marco oportuno del Foro Económico Mundial sobre la ASEAN celebrado en septiembre, tendrá todavía muchos retos por superar.

\section{Vietnam e India}

India y Vietnam firmaron un acuerdo de asociación estratégica integral en 2016 que no había rendido frutos hasta 2018. Este acuerdo se ha reforzado con intercambios de alto nivel entre los dos países. En principio, se tiene la visita a India que realizó el presidente vietnamita Tran Dai Quang en enero, y del primer ministro vietnamita, Nguyen Xuan Phuc, en marzo. Este último se entrevistó con su homólogo, Narendra Modi, y de esa entrevista surgió la solicitud india de facilitar su inversión en los sectores de gas y petróleo, energías renovables, y farmacéutico.

Sin embargo, lo más importante de la relación se dio en materia comercial, cuyo intercambio ha superado los 10 mil millones de dólares, que desean incrementar a 15 mil millones para el 2020. Del sureste de Asia, Vietnam figura en el cuarto lugar como socio comercial de India. 
De igual importancia son los asuntos de seguridad y petróleo. En junio, el ministro de Defensa indio, Nirmala Sitharaman, de visita en Vietnam refirió la importancia del tema de defensa. Por su parte, el viceministro de Defensa vietnamita, Nguyen Chi Vinh, visitó la India para participar en el XI Diálogo de Política de Defensa Vietnam-India, en el Ministerio de Defensa de India (Parameswaran, 2018). Según una entrevista a medios vietnamitas, Chi Vinh expresó que los dos países consensuaron en reforzar su cooperación en Defensa hasta 2019 y más allá. Se comprometieron también a intensificar la ayuda recíproca para el mantenimiento de la paz, la garantía de la seguridad marítima y la cooperación en ciencias y tecnologías militares (VOV5, 2018).

Respecto al asunto del petróleo, el primer ministro Xuan Phuc dio la bienvenida a la Cooperación de Petróleo y Gas Natural (ONGC, por sus siglas en inglés) con la parte india en la exploración y explotación de petróleo en la zona económica exclusiva y la plataforma continental de Vietnam en el Mar del Sur de China. La exploración de petróleo y gas ha sido clave para la cooperación entre ambos países con compañías indias que realizan importantes inversiones en este sector. China, por su parte, se opone a esta cooperación que explora el petróleo en aguas reclamadas por Vietnam. India ha afirmado que la exploración de ONGC es una operación comercial y no está relacionada con la disputa.

Es comprensible que la exploración petrolera en el Mar del Sur de China sea un tema delicado en las relaciones Vietnam-China. El ministro del exterior chino, Lu Kang, mencionó que su país no se opone al desarrollo de relaciones bilaterales de países vecinos, refiriendo a Vietnam, sino a que las partes las utilicen como excusa para infringir los derechos legítimos de China y perjudicar la paz y la estabilidad regionales (Hindustan, 2018).

Otro asunto de la relación bilateral indo-vietnamita fue el compromiso sobre lo que se conoce como economía azul (blue economy). El concepto describe el manejo sostenible de los recursos oceánicos, el mejoramiento de la calidad de vida y de los empleos, y el mantenimiento saludable los ecosistemas oceánicos. ${ }^{3}$ En julio se llevó a cabo el Segundo Taller ASEAN-India sobre economía azul, al que asistió el embajador vietnamita Ton Sinh Thanh, quien expresó su esperanza de que pronto se concluyeran las negociaciones sobre el

\footnotetext{
${ }^{3}$ El término blue economy fue llevado a la mesa de negociación en un encuentro en Indonesia entre el primer ministro de India, Narendra Modi, y el presidente de Indonesia, Joko Widodo, en 2017.
} 
acuerdo de transporte marítimo ASEAN-India. Corresponde a la misma agenda de seguridad entre ambos países.

La secretaria de la sección este del Ministerio de Asuntos Exteriores de India, Preeti Saran, afirmó que su país destinaría 190 mil millones de dólares para impulsar la conectividad entre sus puertos marítimos, y 27 nuevas áreas de desarrollo económico. Invitó a los países de la ASEAN a considerar unirse al proyecto, centrándose inicialmente en zonas económicas especiales costeras, consultas sobre inversiones, transporte marítimo y turismo (Vietnam, 2018).

\section{Vietnam y Australia}

Vietnam y Australia actuaron de manera proactiva y no pasiva como lo habían estado haciendo años atrás. Un antecedente en 2009 inició con el acuerdo de una asociación integral, seguida de una asociación integral avanzada en 2015 que culminó con la puesta en marcha del Plan de Acción Australia-Vietnam 2016-2019, en el marco de una asociación estratégica para la paz, la cooperación y la prosperidad en Asia-Pacífico y el mundo. De este modo se anunció en 2018, con motivo de la conmemoración de los 45 años de relación (Embassy, 2018).

Destaca también el informe de los recursos ejercidos por la asistencia oficial australiana para el desarrollo de Vietnam, en los siguientes términos: para el ejercicio 2017-2018 se estimó una colaboración de Australia por 82.2 millones de dólares australianos (AOD, 2018). Los objetivos hacia donde han sido dirigidos los montos están incluidos en el Plan de Inversión de Ayuda 2015-2020. Destacan, entre otros, involucrar al sector privado, desarrollo y empleo de fuerza laboral altamente calificada, y empoderamiento económico de la mujer. Hay otros programas de asistencia relacionados con: becas; promoción de vínculos industriales con la educación; infraestructura, como el puente Cao Lanh concluido en mayo pasado, y acceso a agua corriente en comunidades empobrecidas (AOD, 2018).

En suma, la política exterior del gobierno de Vietnam en 2018 estuvo encaminada a mantener las relaciones con el mayor número posible de países, en tanto fortaleció otras y emprendió nuevas con países de otras latitudes. Estados Unidos, China, India y Australia fueron los lazos más distinguidos. Con Japón, Singapur y la Comunidad Europea sigue fortaleciéndose. 


\section{Entorno económico interno}

Parte del impulso económico de Vietnam proviene de los centros urbanos, Ho Chi Minh y las provincias circundantes, incluida Ba Ria-Vung Tau con su desarrollo portuario. Asimismo, hay puntos en alto desarrollo, como la capital, Hanói, la ciudad portuaria Haiphong, Hue, Danang, y Can Tho. Todos estos puntos en desarrollo están estratégicamente ubicados por todo el país. En algunas provincias se albergan centros industriales importantes, en particular los de inversión extranjera; por eso dichas ciudades tienen altas tasas de fuerza laboral.

Vietnam tiene actualmente tres parques de alta tecnología, ubicados en Hanói, Da Nang y Ho Chi Minh, que han atraído más de 200 proyectos de inversión, con un capital registrado de casi 10 mil millones de dólares. Tal resultado ha sido una base importante para optimizar las potencialidades científico-tecnológicas del país y contribuir al desarrollo socioeconómico en las localidades y regiones (Van, 2018).

Desde enero de 2018, el primer ministro vietnamita, Nguyen Xuan Phuc, dejó clara la necesidad de desarrollo del campo como estrategia para el crecimiento rural. Reconociendo que 2017 fue de bajo rendimiento agrícola, urgió a "desarrollar la producción masiva,

promover la aplicación de la alta tecnología y aprovechar las oportunidades de la Cuarta Revolución Industrial para elevar el rendimiento, la eficacia y la competitividad. La construcción de la nueva ruralidad tiene que acompañarse con el mejoramiento de la productividad y la vida material y espiritual de los pobladores" (Vov5, 2018). Sin embargo, a decir de Xuan Phuc, éstas no son importantes sólo para 2018, sino también para los años siguientes.

A continuación se analiza el comportamiento económico de algunos sectores relevantes en 2018.

\section{Pesca}

De acuerdo con la Oficina General de Estadística de Vietnam (GSO, 2018), el sector agrícola ha tenido buenos resultados en lo que va del año, y el subsector más sobresaliente ha sido el pesquero. Se estima que la producción pesquera de enero a agosto de 2018 alcanzó 4929.1 
miles de toneladas, 5.8\% más que en el mismo periodo de 2017, incluidas la producción acuícola y la pesca.

La cría de camarón se está convirtiendo en una actividad de alta calidad de exportación, cuyos principales compradores son la Unión Europea y Estados Unidos. De hecho, Vietnam es considerado en este momento el tercer gran exportador de producción acuícola, con la del camarón a escala mundial. El aspecto negativo de esta actividad es que es tan rentable, que pequeños agricultores han cambiado su actividad en el campo por la acuicultura, lo cual crea la necesidad de talar los manglares para el cultivo de camarón, con el consecuente irreparable daño ambiental.

Tardíamente, compañías de alimentos realizan esfuerzos para utilizar medios de producción menos agresivos. En el Delta del Mekong, parte sur de Vietnam, algunos pescadores trabajan en la reforestación de manglares para volver a su actividad. La diferencia es que hoy obtendrán un certificado de producto orgánico, con valor agregado, en cuanto cumplan con los estándares de sustentabilidad y cuidado del medio ambiente. Ésta es una iniciativa de organizaciones no gubernamentales, extranjeras, apoyadas por el gobierno central.

\section{Inversión}

En los primeros ocho meses de 2018 la inversión de capital estatal llegó a 183.6 billones de dong, un aumento de $11.3 \%$ en relación con el año anterior. En el mismo periodo, la IED atrajo 918 proyectos con nueva licencia y un capital total de 13481.6 millones de dólares, lo que representa un incremento de $18.1 \%$ más que el de 2017. Se estima que la IED alcanzada en los ocho primeros meses del año es de 11.25 mil millones de dólares, lo que representa $9.2 \%$ más que en igual periodo del año pasado. Asimismo, de enero a agosto 52 provincias y ciudades administradas de manera centralizada en todo el país recibieron proyectos de inversión extranjera directa, recientemente licenciados de 66 países y territorios (GSO, 2018).

Uno de los sectores que pueden beneficiarse si se formaliza el CPTTP es el textil y prendas de vestir, que atrajo 2800 millones de dólares como IED durante el primer semestre de 2018, con lo que el total en el sector ascendió a casi 17500 millones de dólares (VietnamNet, 2018). De los proyectos a gran escala puestos en marcha, hay uno que incluye 
hilados de lana de oveja con inversión alemana (en la provincia de Da Lat), y una planta de producción de hilados (en Dong Nai) (VietnamNet, 2018).

En otro orden, pero relacionado con la captación de IED al país, está el impulso económico de Vietnam a los corredores con puntos clave para el desarrollo. Muy importantes son el crecimiento de los corredores industriales en centros urbanos de ciudades como Ho Chi Minh y provincias circundantes, como Ba Ria-Vung Tau, pues estas dos ciudades también pueden considerarse corredores entre Hánoi y la ciudad portuaria de Háiphong, o bien la ruta costera de Hue a Danang, o la ruta de Ho Chi Minh a Can Tho; todas, vías de acceso claves e importantes distribuidas por todo el país, que cuentan con importantes montos de inversión extranjera directa.

Las dos primeras rutas de centros urbanos albergan a la mayoría de las industrias que se mueven con IED, cuyos frutos son altas tasas de fuerza laboral.

Según el primer ministro vietnamita, el mayor obstáculo para seguir fomentando estos complejos es institucional, por lo que urgió a los ministerios y sus ramas a promulgar cuanto antes las circulares de orientación relacionadas con las políticas vigentes, y a proponer las enmiendas a algunos artículos de la Ley de Alta Tecnología 2008. Al respecto, Nguyen Xuan Phuc, resaltó la necesidad de incluir tres aspectos en torno a la IED. Primero, deben concretarse políticas que faciliten la atracción de inversión de calidad; segundo, deben recogerse opiniones y sugerencias por parte de inversionistas y presentarlas al gobierno, y tercero, deben trabajar en conjunto los ministerios de Finanzas, Planificación e Inversión y Ciencia-Tecnología, para resolver los obstáculos. "Ésta es una tarea muy importante para lograr el objetivo de construir un ecosistema empresarial en Vietnam” (Van, 2018).

\section{Importación y exportación}

El 1 de enero de 2018 entró en vigor la Ley de Gestión del Comercio Exterior, que incluye medidas específicas para fortalecer la eficiencia administrativa del Estado en las actividades de importación y exportación.

Se estima que durante los primeros ocho meses de 2018 las ventas de bienes de exportación alcanzaron los 155.41 mil millones de dólares, un aumento de $14.5 \%$ en comparación con el mismo periodo de 2017, de los cuales el sector económico nacional 
alcanzó 45.11 mil millones de dólares, esto es, 17.4 \% más. Por su parte, la IED (incluido el petróleo crudo) ganó 110.3 mil millones de dólares (que representan $71 \%$ del total de exportaciones), 13.4 por ciento más.

Acerca de la exportación de mercancías, Estados Unidos fue en ocho meses el mayor mercado de Vietnam con una facturación de 30.2 mil millones de dólares, 10.2\% más que en el mismo periodo del año anterior; seguido por la UE, con 27.7 mil millones de dólares, un aumento de $10.8 \%$, de los cuales la electrónica, las computadoras y sus partes crecieron 23.3\%; China, con 23.4 mil millones y un incremento de 25.2\%; ASEAN, con 16.5 mil millones, 16\% más; Japón, con 12 mil millones, subió 9.4\%; Corea del Sur, con 11.8 mil millones y un crecimiento de 27.9 por ciento.

En general, en ocho meses los volúmenes de importación de mercancías alcanzaron un estimado de 152.66 mil millones, $11.6 \%$ más que en el mismo periodo del año anterior, de los cuales el sector económico nacional ganó 61.85 mil millones, $11.8 \%$ más; el sector de IED alcanzó 90.81 mil millones, esto es, un incremento de 11.4 por ciento.

En cuanto a la importación de mercancías en ocho meses, China sigue siendo el mayor mercado de importación de Vietnam, con una facturación de 41.4 mil millones, 12.8\% más respecto al correspondiente periodo de 2017; seguido por Corea del Sur, con 30.8 mil millones, 1.9\% más; ASEAN, con 20.5 mil millones, un incremento de $11.8 \%$; Japón, con 12.2 mil millones y aumento de $14.8 \%$; UE, con 8.9 mil millones, esto es, un crecimiento de 11.2\%; EUA, con 8.7 mil millones, 39.2 por ciento arriba (GSO, 2018).

La balanza comercial de mercancías realizada en julio tuvo un déficit comercial de 635 millones de dólares. Según lo estimado, el déficit comercial fue de 100 millones de dólares en agosto. En general, en ocho meses la balanza comercial mantuvo un superávit comercial con 2.8 mil millones de dólares, de los cuales el sector económico interno tuvo un déficit comercial de 16.7 mil millones, y la IED (incluido el petróleo crudo) un superávit comercial de 19.500 mil millones de dólares (GSO, 2018).

\section{Visitantes internacionales}

Las estadísticas oficiales muestran signos positivos para la industria turística y confirman que el número de visitantes a Vietnam va en aumento. Reportan además que de enero a agosto 
se dieron 10403000 arribos, lo que representa un aumento de $22.8 \%$ en comparación con 2017. La procedencia de los visitantes fue principalmente de Asia (con 8034 000), Europa (con 1404 000) y América (con 636 000) (GSO, 2018).

Por provincia, fuera de las ciudades clásicas como Ho Chi Minh o Hanói, la más visitada fue Binh Thuan, en la costa central del país, que recibió a más de cuatro millones de turistas en el primer cuatrimestre. Las inversiones pública y privada que se inyectan en este país, en la rama turística, se reflejan con creces si se observa la culminación de varios megaproyectos no hoteleros, como el nuevo Puente de Oro (Golden Bridge), cuyo significado cultural lo vuelve un sitio que necesariamente debe visitarse si se está en Vietnam. Se trata de un puente situado entre las colinas de $\mathrm{Ba} \mathrm{Na}$, cuya estructura simula una pasarela sostenida por unas grandes manos. Se eleva unos 1000 metros (3 280 pies) sobre el nivel del mar, y se extiende sobre las copas de los árboles, enmedio de un acantilado. Fue inaugurado en junio de 2018.

De los aciertos que han motivado el turismo en Vietnam, su bajo costo es uno de los mayores atractivos. Un ejemplo es la ciudad antigua de Hoi An, clasificada como el quinto destino más barato del mundo (Telegraph, 2018). Pero además, Hoi An es patrimonio mundial, nombrado por la UNESCO, recientemente clasificado como uno de los 10 destinos más seguros para personas que viajan solas, y está ubicado entre las 15 mejores ciudades del mundo (Walhout, 2018).

\section{Temas sociales}

La escasez de alimentos ha incrementado el número de familias con este padecimiento durante 2018, y se ha vuelto un tema muy importante a considerar en todo el país, toda vez que tiene impacto directo en los índices de pobreza. Sin embargo, la disminución gradual de la pobreza le ha merecido un reconocimiento en el ámbito internacional. El escaso rendimiento productivo de productos agrícolas, aves y ganado, fue resultado de las graves inundaciones sucedidas a finales de 2017 y principios del actual. Como responsables de apoyar a los damnificados de las inundaciones, quedaron varios organismos administrativos, sectores e instituciones de los niveles central y local. El apoyo económico fue de aproximadamente 7.4 millones de toneladas de granos y 1.6 mil millones de dong (gso, 2018). 
Entre otros temas sociales, como ya se dijo, se suscitaron manifestaciones populares en contra de la nueva ley de arrendamiento de tierras para extranjeros, en contra de la creación de zonas económicas especiales y en contra de la nueva ley de seguridad de internet, entre otras que obligaron al despliegue policial en las principales ciudades del país.

El luto también se presentó en Vietnam. La muerte de su presidente, Tran Dai Quang, a los 61 años, tomó por sorpresa a la sociedad vietnamita porque su presencia en foros internacionales y su trabajo político fueron bien conocidos. En agosto hizo visitas oficiales a Egipto y a Etiopía. El pésame acercó a los líderes que se han mencionado en este artículo: Donald Trump, Vladimir Putin y Xi Jinping, entre otros. Murió el 21 de septiembre.

En su lugar y por primera vez el puesto de presidente fue ocupado por una mujer, Danh Thi Ngoc Thinh, quien fungía como vicepresidenta. Su cargo durará hasta que la Asamblea Nacional convoque a una sesión especial para elegir al nuevo presidente, y deberá conocerse tan pronto como se organicen los funcionarios. Cabe agregar que es la primera mujer en ocupar este cargo en la historia de Vietnam, y la primera en los países comunistas.

En términos generales, 2018 fue un año exitoso para Vietnam, pues mantuvo su prestigio internacional y regional ganado a pulso y que lo han caracterizado por varios años. Posiblemente ha tenido que repensar sus estrategias en cuanto a relaciones bilaterales, principalmente con Estados Unidos y China, lo que no le resta importancia al hecho de que sigue ampliando sus relaciones con otros países. Seguramente tendrá nuevos retos que enfrentar mientras resuelve el del Mar del Sur de China. Pero el mayor éxito lo tiene en el terreno económico, pues incluso lo llaman el nuevo tigre de Asia debido a su crecimiento económico sostenido de entre 6 y 7\%, que no es fácil de lograr. Sin embargo debe resolver cómo volverse atractivo, en términos comerciales, para el mundo entero.

\section{REFERENCIAS BIBLIOGRÁFICAS}

AOD (2018), Australian Government, Department of Foreign Affairs and Trade. Recuperado de https://dfat.gov.au/geo/vietnam/development-assistance/Pages/developmentassistance-in-vietnam.aspx

Beech, H. (2017), The New York Times, 11 de noviembre. Recuperado de https://www.nytimes.com/2017/11/11/world/asia/vietnam-china-us.html 
Beech, H. (2018), New York Times, 4 de marzo. Recuperado de https://www.nytimes.com/2018/03/04/world/asia/carl-vinson-vietnam.html

Dang, D. N. (2018). The Diplomat, 3 de mayo. Recuperado de https://thediplomat.com/2018/03/loving-vietnam-criticizing-cambodia-trumpsdouble-standard-in-southeast-asia/

Embassy, A. (2018), Australian Embassy, 3 de mayo. Recuperado de https:/vietnam.embassy.gov.au/hnoi/MR180117.html

Fawthrop, T. (2018), The Diplomat, 21 de junio. Recuperado de https://thediplomat.com/2018/06/vietnam-mass-protests-expose-hanois-chinadilemma/

Gao, C. (2018), The Diplomat, 18 de abril. Recuperado de https://thediplomat.com/2018/04/ahead-of-drills-in-south-china-sea-chinese-foreignminister-visits-vietnam/

GSO (2018), General Statistics Office of Vietnam. Recuperado de http://www.gso.gov.vn/default_en.aspx?tabid=622\&ItemID=18928

Hindustan, (2018), Hindustan Times, 11 de enero. Recuperado de https://www.hindustantimes.com/world-news/china-slams-vietnam-for-inviting-indiato-invest-in-south-china-sea/story-WuNr8npFE6giZLaUgKsWXK.html

Hutt, D. (2018), Asia Times, 9 de julio. Recuperado de http://www.atimes.com/article/innod-to-north-korea-pompeo-praises-vietnam/

Maliszewska, M., Z. Olekseyuk, e I. Osorio-Rodarte (2018), World Bank Group, 5 de marzo.

Recuperado

de http://documents.worldbank.org/curated/en/530071520516750941/Economic-anddistributional-impacts-of-comprehensive-and-progressive-agreement-for-transpacific-partnership-the-case-of-Vietnam

Panda, A. (2018), The Diplomat, 28 de mayo. Recuperado de https://thediplomat.com/2018/05/south-china-sea-two-us-navy-destroyers-conductfreedom-of-navigation-operation-in-paracel-islands/ 
Parameswaran, P. (2018), The Diplomat, 3 de agosto. Recuperado de https://thediplomat.com/2018/08/whats-next-for-vietnam-india-defense-relations/

State, U. D. (2018), U.S. Department of State, 20 de junio. Recuperado de https://www.state.gov/r/pa/prs/ps/2018/06/283374.htm

Suiwah, L. (2018), East Asia Forum, 19 de septiembre. Recuperado de http://www.eastasiaforum.org/2018/09/19/can-vietnam-weather-trumps-trade-storm/

Telegraph, T. (2018), The Telegraph Travel News, 14 de septiembre. Recuperado de https://www.telegraph.co.uk/travel/news/post-office-long-haul-cape-town-cheapestwinter-sun/

Van, D. (2018), vov La Voz de Vietnam, 26 de febrero. Recuperado de http://vovworld.vn/esES/enfoque-de-actualidad/vietnam-acelera-reformas-economicas-para-lograr-uncrecimiento-de-mayor-calidad-622299.vov

Vietnam (2018), 18 de julio. Recuperado de https://en.vietnamplus.vn/second-aseanindiaworkshop-on-blue-economy-held/134893.vnp

Vietnam, V. T. (2018), 14 de septiembre. Recuperado de https:/english.vov.vn/economy/foreign-media-vietnam-japan-call-on-us-to-rejoincptpp-383312.vov

VietnamNet (de 2018), 18 de marzo. Recuperado de https://english.vietnamnet.vn/fms/business/206294/cptpp--evfta-help-vietnam-sgarment-textile-lure-investment.html

VietnamPlus (2018), Funcionarios estadounidenses elogian relaciones de asociación integral de su país con Vietnam, 28 de mayo. Recuperado de https://es.vietnamplus.vn/funcionarios-estadounidenses-elogian-relaciones-deasociacion-integral-de-su-pais-con-vietnam/88511.vnp

vov5 (2018), 2 de agosto. Recuperado de http://vovworld.vn/es-ES/noticias/vietnam-y-laindia-por-reverdecer-la-confianza-mutua-en-defensa-666925.vov 
vov5 (2018). La Voz de Vietnam, 15 de junio. Recuperado de http://vovworld.vn/esES/noticias/vietnam-determinado-a-cumplir-la-convencion-de-la-onu-sobre-elderecho-del-mar-de-1982-653247.vov

vov5 (2018), VOV5 La Voz de Vietnam Voz del Mundo, 4 de enero. Recuperado de http://vovworld.vn/es-ES/noticias/premier-vietnamita-orienta-las-metas-para-elsector-agricola-nacional-608663.vov

Walhout, H. (2018), Travel and Leisure, 10 de julio 7. Recuperado de https://www.travelandleisure.com/worlds-best/cities-in-asia

WEFORUM-ASEAN (s.f.). World Economic Forum on ASEAN. Recuperado de https://www.weforum.org/events/world-economic-forum-on-asean 